\title{
ESTIMATION OF MEASUREMENT UNCERTAINTY WITH THE USE OF UNCERTAINTY DATABASE CALCULATED FOR OPTICAL COORDINATE MEASUREMENTS OF BASIC GEOMETRY ELEMENTS
}

\author{
Danuta Owczarek ${ }^{1}$, Ksenia Ostrowska ${ }^{1}$, Wiktor Harmatys' ${ }^{1}$, Jerzy Sładek ${ }^{1}$ \\ 1 Laboratory of Coordinate Metrology, Faculty of Mechanical Engineering, Cracow University of Technology, \\ al. Jana Pawła II 37, 31-864 Kraków, Poland, e-mail: dszewczyk@mech.pk.edu.pl; kostrowska@mech.pk.edu. \\ pl;wharmatys@mech.pk.edu.pl; sladek@mech.pk.edu.pl
}

Received: 2015.05.13

Accepted: 2015.08.05 Published: 2015.09.01

\begin{abstract}
The paper presents a method for estimating the uncertainty of optical coordinate measurement based on the use of information about the geometry and the size of measured object as well as information about the measurement system, i.e. maximum permissible error (MPE) of the machine, selection of a sensor, and also the required measurement accuracy, the number of operators, measurement strategy and external conditions contained in the developed uncertainty database. Estimation of uncertainty is done with the use of uncertainties of measurements of basic geometry elements determined by methods available in the Laboratory of Coordinate Metrology at Cracow University of Technology (LCM CUT) (multi-position, comparative and developed in the LCM CUT method dedicated for non-contact measurements) and then with the use of them to determine the uncertainty of a given measured object. Research presented in this paper are aimed at developing a complete database containing all information needed to estimate the measurement uncertainty of various objects, even of a very complex geometry based on previously performed measurements.
\end{abstract}

Keywords: estimation of measurement uncertainty, optical coordinate measuring systems

\section{INTRODUCTION}

Together with growing importance of optical coordinate measuring systems, and expanding area of their applications grows the need to systematize the issue of uncertainty of measurements done with the use of them. The measurement result given without information about the uncertainty of its obtaining, from a technical point of view, has of little importance. The issue of measurement uncertainty is constantly tested and improved in relation to needs of machines and instruments market development [1-6].

To face problems associated with the measurement uncertainty issue especially in industrial practice in the Laboratory of Coordinate Metrology have been designed a cycle of studies aimed at developing advanced database of uncertainties together with the methodology of its effective use. Uncertainties included in the database are provided from measurements of profiles surfaces models produced in a technology of Fused Deposition Modeling (FDM) with the use of machine developed in the LCM CUT, which are then used for estimating the uncertainty of a real measurement. The measurement uncertainty is estimated basing on similarities with the uncertainty database in case of following factors:

- geometry, size of measured object,

- MPE - maximum permissible error of the machine,

- required measurement accuracy,

- sensor type,

- measurement strategy,

- the number of operators,

- external conditions (especially temperature).

At the present stage of research, optical coordinate measuring systems available in the Labo- 
ratory of Coordinate Metrology and elements of basic geometry are used to verify the correctness of taken assumptions. The use of uncertainty database significantly reduces the time needed to obtain the uncertainty for real measurement, and what's more it allows the estimation of uncertainty in situations when it is not possible - for example because of lack of appropriate standards.

\section{SYSTEMS AND MODELS}

\section{Optical coordinate measuring systems}

Research with the use of optical coordinate measuring systems available in the Laboratory of Coordinate Metrology are aimed at possessing data from measurements of printed models of geometrical elements. The above-mentioned systems are presented in Figure 1 Coordinate Measuring Machine (CMM) LK V10.7.6 SL of Nikon Metrology, Articulated Arm Coordinate Measuring Machine (AA CMM) 73SI (both CMM and AA CMM are equipped with optical probe heads) and optical system SMARTTECH 3D - SCAN3D qualify 10 Mpi operating by white light. Measurements are carried out in accordance with procedures developed on the basis of standards (among others [7-10]) and many years of experience of the LCM team. In the next step appropriate measurement uncertainties are determined and with the use of them developed database is composed.

\section{Measured object models}

To make maximally complete measurement an uncertainty database is possible to be created without generating significant costs associated with the purchase of special standards, to create models of geometric elements the Fused Deposition Modelling (FDM) technology belonging to the group of rapid prototyping methods is use. This technology is mainly used for 3D printing (modelling, prototyping). It operates by laying down material in layers in that case a plastic filament. In the Laboratory of Coordinate Measurements for production of models uses a printer of $500 \times 500 \times 500 \mathrm{~mm}$ workspace, constructed there. Because of it a broad measuring base composed of among other elements is obtained quickly. They included the following ones:

- sphere,

- bowl,

- external/internal cylinder,

- external cone,

- internal cone,

- sinusoidal surface (Fig. 2),

- internal step,

- external step,

- plane.

\section{Database use}

The purpose of the developed database is to allow the operator the estimation of the uncertain-

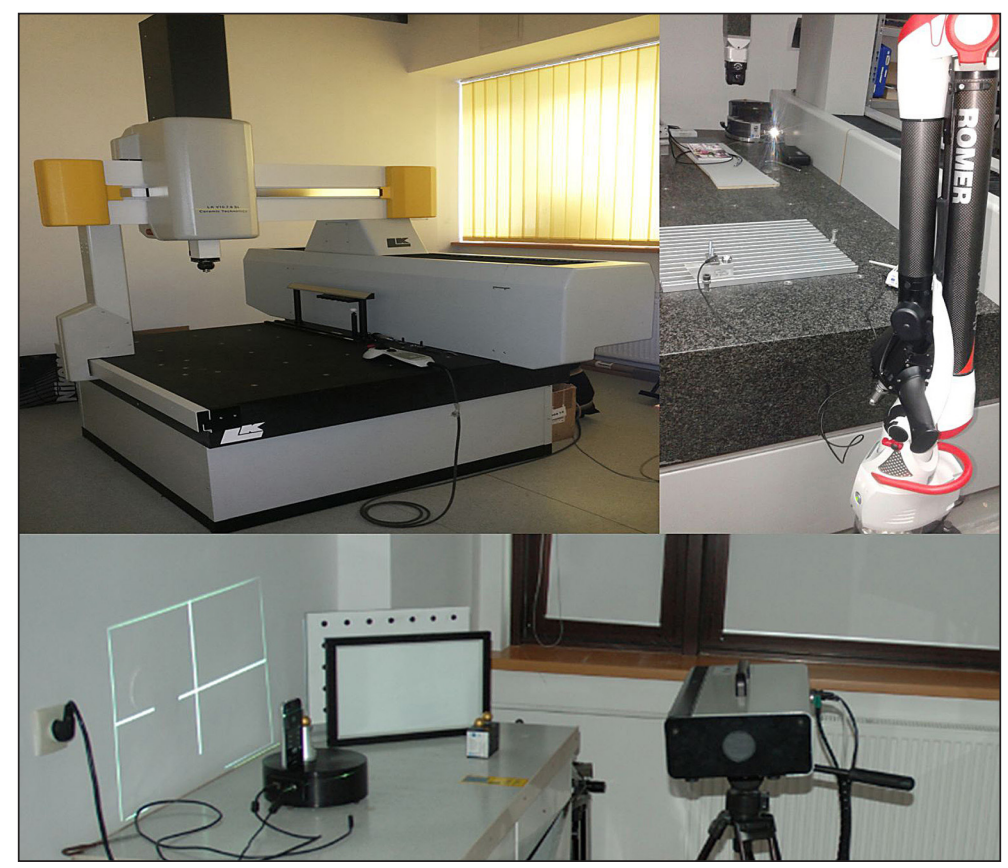

Fig. 1. Coordinate Measuring Machine LK V10.7.6 SL - on the left side, Articulated Arm Coordinate Measuring Machine 73SI - on the right side, optical system SMARTTECH 3D - SCAN3D qualify $10 \mathrm{Mpi}-$ on the bottom 


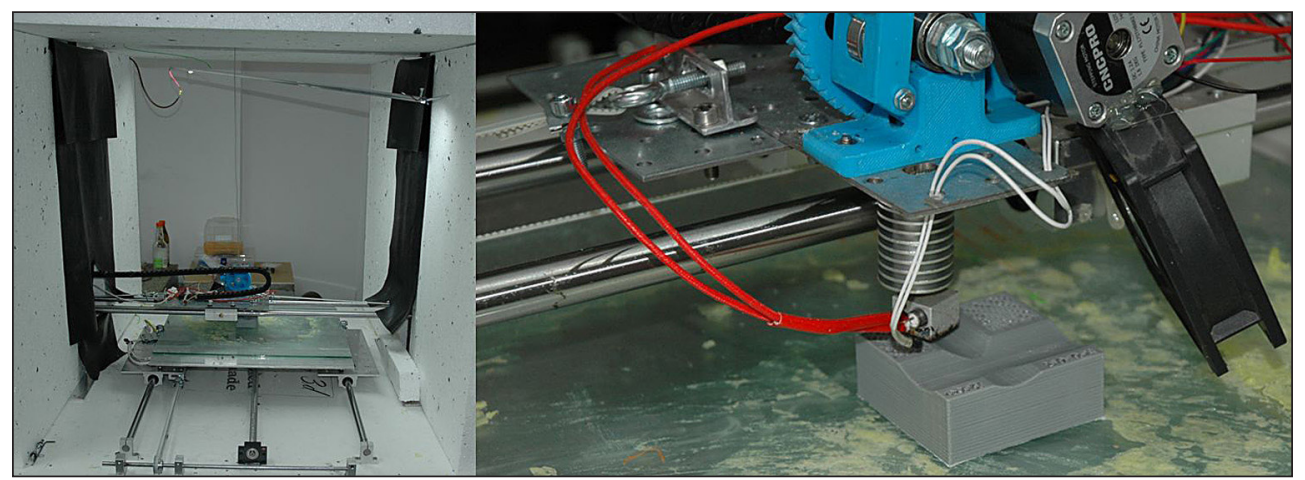

Fig. 2. The process of sinusoidal surface standard projecting process with the use of FDM technology on a machine modelled in the LCM

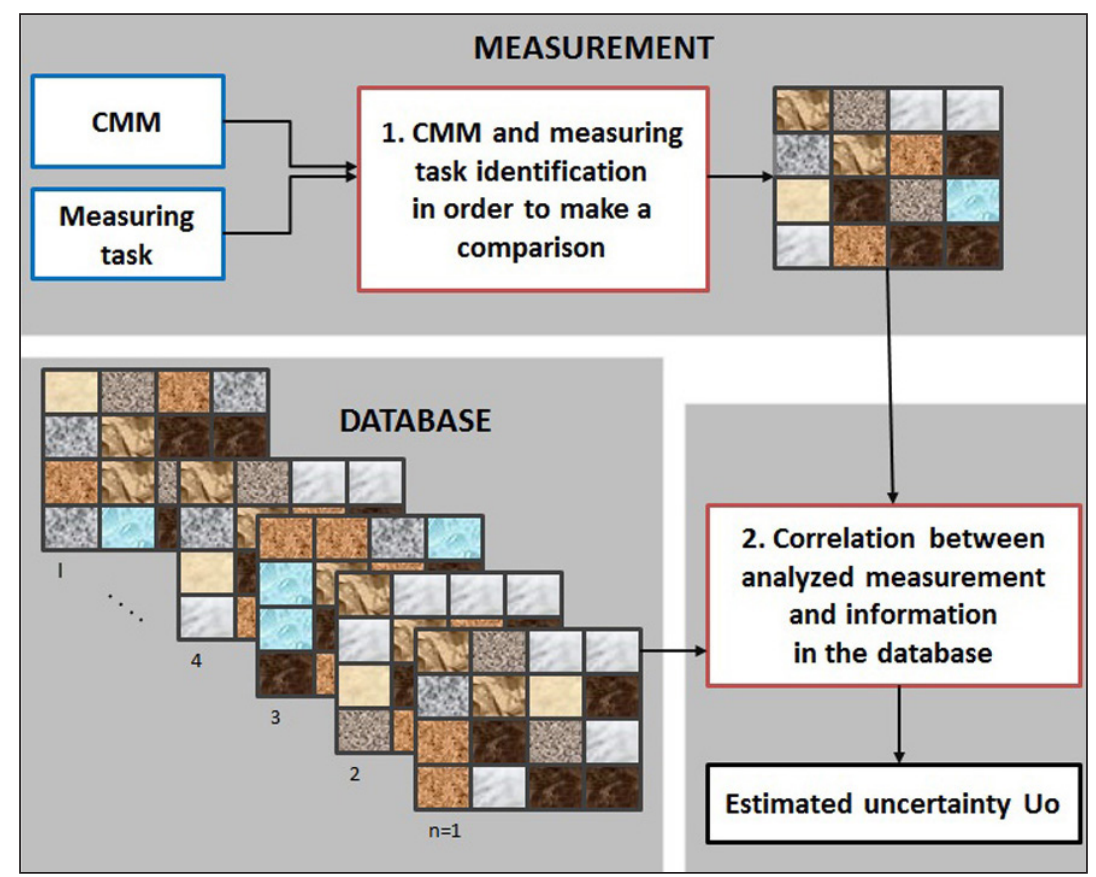

Fig. 3. The basic scheme of measurement uncertainty database method based on EXPERT system proposed by Hansen H.N. [14]

ty of realized measuring task by the use of data collected in it. The basic principle of the measurement uncertainty database operation scheme is presented in Figure 3.

The database stores information about the coordinate measuring system and measuring task together with corresponding uncertainties. Each record in the database refers to a particular machine and to a particular measurement performed with the use of this machine together with the uncertainty determined for this measurement. Currently, the records are carried out work to improve the selection algorithm for typing of corresponding to the real measurement information from the database and for estimating the measurement uncertainty based on them. The comparison results with the correlation coefficients for each of found information in the database to performed measurement (the degree of a compliance). Finally, estimated measurement uncertainty is calculated as a weighted mean value from uncertainties for which similarities with the real measurement have been detected [15].

\section{METHODS}

In research three methods for determining the measurement uncertainty were used:

- Multi-position method,

- Comparative method,

- OPTI-U method (Optical Uncertainty) - developed in the Laboratory of Coordinate Metrology dedicated for determining the uncertainty of optical coordinate measurements. 


\section{Multi-position method}

Multi-position method involves using a variety of factors, combining the use of the non-calibrated measuring object with frequently repeated measuring strategy $[11,12]$.

$$
\begin{gathered}
\mathrm{U}=\left|E_{L}\right|+\left|E_{D}\right|+ \\
k \sqrt{\left(u_{\text {rep }}\right)^{2}+\left(u_{\text {geo }}\right)^{2}+\left(u_{\text {corr }}\right)^{2}+\left(u_{D}\right)^{2}+\left(u_{\text {temp }}\right)^{2}}
\end{gathered}
$$

where: $E_{L}-$ component of error of length measurement $[\mathrm{mm}], \mathrm{E}_{\mathrm{D}}$ - component of average error for external or internal diameter measurement or for flat surface [mm], $\mathrm{k}$ - extension factor depended on effective degrees of freedom, $u_{\text {rep }}$ - standard uncertainty component depended on CMM repeatability $[\mathrm{mm}], \mathrm{u}_{\mathrm{geo}}-$ standard uncertainty component depended on CMM geometrical errors [mm], $\mathrm{u}_{\text {corrL }}-$ correction uncertainty, included in length measurement of the standard $[\mathrm{mm}], \mathrm{u}_{\mathrm{D}}$ - standard uncertainty of difference of errors between internal and external standard measurements [mm], $\mathrm{u}_{\text {temp }}$ - standard uncertainty depended on temperature of measured object, standard and environment $[\mathrm{mm}]$.

\section{Comparative method}

The comparative method involves making measurements of the calibrated standard of a form and size closed to the measured object $[11,12]$.

$$
\mathrm{U}=k \sqrt{\left(u_{c}\right)^{2}+\left(u_{p}\right)^{2}+\left(u_{w}\right)^{2}+\left(u_{b}\right)^{2}}
$$

where: $\mathrm{k}$ - extension factor depended on effective degrees of freedom, $u_{c}-$ uncertainty component depended on standard [mm], $\mathrm{u}_{\mathrm{p}}$ - uncertainty component depended on the used procedure $[\mathrm{mm}], \mathrm{u}_{\mathrm{w}}-$ uncertainty component depended on measured object $[\mathrm{mm}], \mathrm{u}_{\mathrm{b}}-$ uncertainty component depended on systematic error [mm].

\section{OPTI-U method}

OPTI-U method is a method developed in the Laboratory of Coordinate Metrology with the partly use of components of multi-position method, and of methods known in the metrology as R\&R [1]. It is aimed at determining the uncertainty of optical coordinate measurements [13].

$$
\begin{gathered}
\mathrm{U}=\left|E_{L}\right|+\left|E_{D}\right|+\left|E_{R}\right|+\left|E_{S}\right|+ \\
k \sqrt{\left(u_{A V}\right)^{2}+\left(u_{E V}\right)^{2}+\left(u_{C A L}\right)^{2}+\left(u_{C T E}\right)^{2}}
\end{gathered}
$$

where: $E_{L}-$ component of error of length measurement, $\mathrm{mm}$,

$E_{D}$ - component of average error for external or internal diameter measurement or for a flat surface [mm],

$\mathrm{E}_{\mathrm{PR}}-$ component of used software [mm], $\mathrm{E}_{\mathrm{S}}$ - component of matching of ,points clouds" [mm],

$\mathrm{k}$ - extension factor depended on effective degrees of freedom, $\mathrm{u}_{\mathrm{AV}}$ - uncertainty component depended on machine operator [mm], $\mathrm{u}_{\mathrm{EV}}$ - uncertainty component depended on gauge $[\mathrm{mm}]$, $\mathrm{u}_{\mathrm{CAL}}$ - uncertainty component depended on calibration value $[\mathrm{mm}]$, $\mathrm{u}_{\mathrm{CTE}}-$ uncertainty component depended on calibration temperature [mm].

\section{RESULTS}

The measurement was carried out with the use of coordinate measuring machine LK V10.7.6 SL of Nikon Metrology company equipped with a linear scanner LC60Dx. A measurement object was the internal and external diameter of the cylinder (Fig. 4a). As a standard a reference ring of a diameter of $28 \mathrm{~mm}$ was used (Fig. 4b), and as a reference measurement, the results from cylinder and standard measurements with the use of Zeiss machine were used (Fig. 4c).

Data in the form of point clouds were developed in Focus 10 program, with the use of filtration methods available in the program (Fig. 5). For developing the results, all methods for determining of measurement uncertainty presented in chapter 2 (Multi-position, comparative and developed in the LCM CUT method dedicated for non-contact measurement - OPTI-U) were used (Table 1).

Presented results show the impact of the used measurement method on the measurement result and on the uncertainty of its obtaining. During measurement, equal filters (in the software dedicated to optical measurements) and equal measurement conditions were used. To make the more extensive commentary, all stages of research have to be complete. Obtained uncertainties are used to create a part of the database for analogous measurements. 


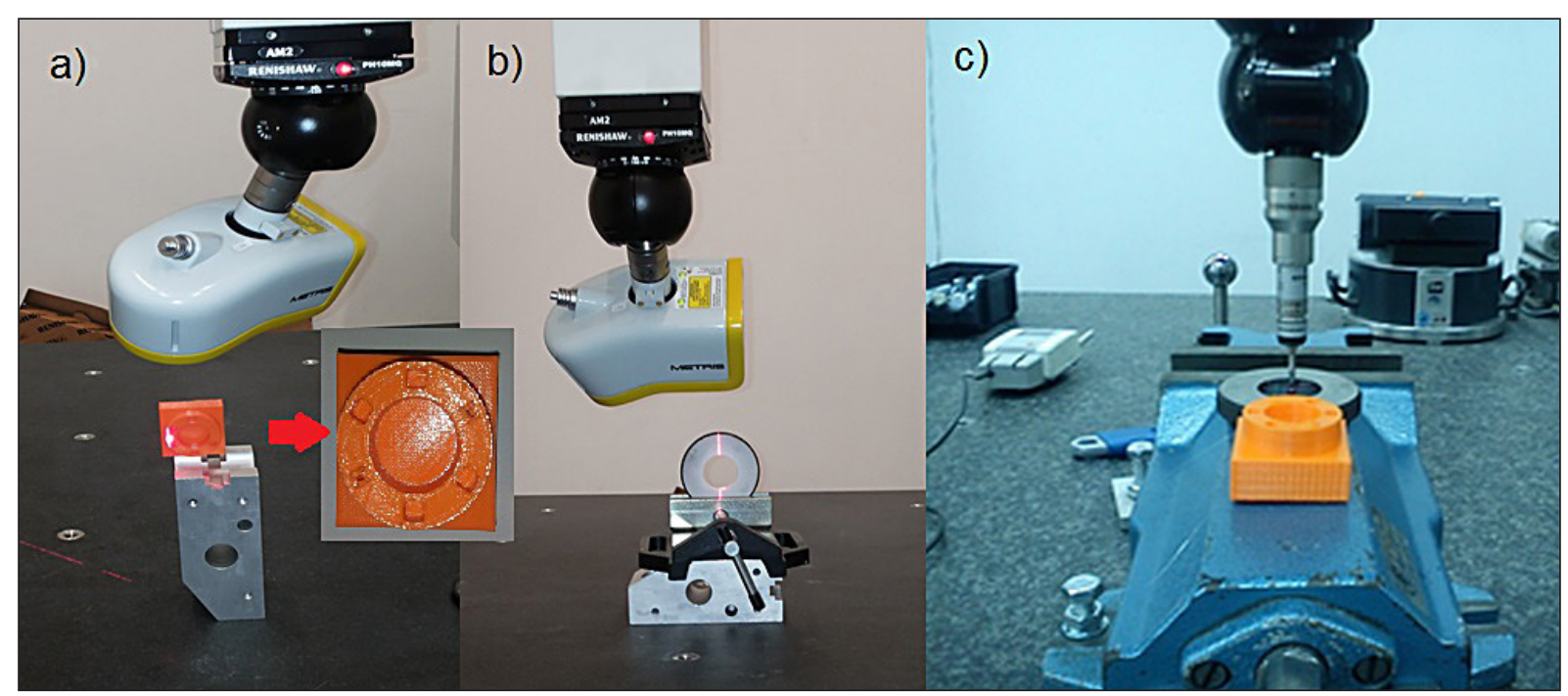

Fig. 4. Measurement carried out with the use of coordinate measuring machine LK V10.7.6 SL of Nikon Metrology company equipped with a linear scanner LC60Dx a) measurement of the object b) measurement of the standard, c) the reference measurement of the object and the standard with the use of Zeiss machine

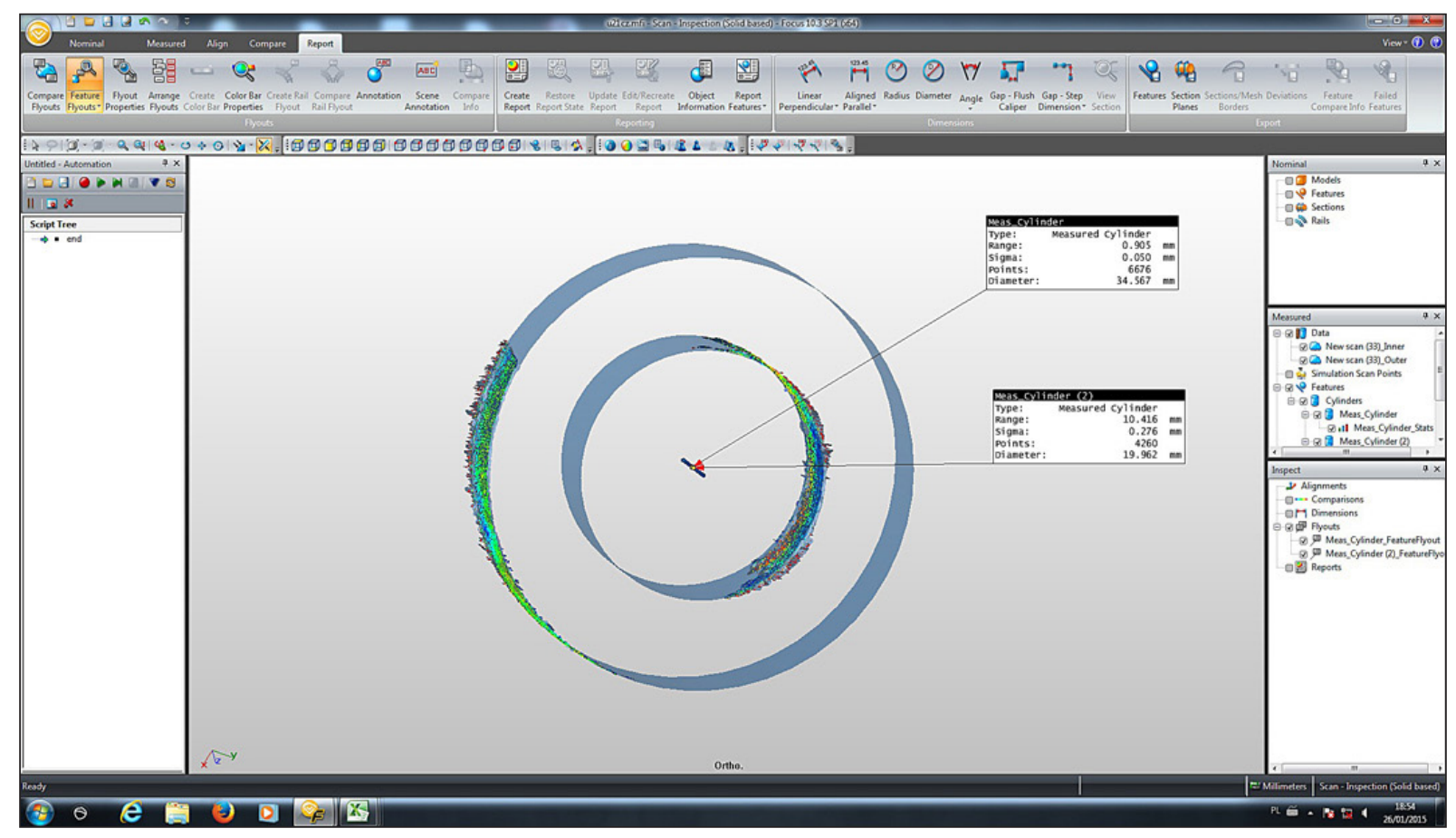

Fig. 5. Reports generating in Focus program

Table 1. Results of measurements performed with the use of optical probe head with uncertainties developed with methods used in the LCM

\begin{tabular}{|c|c|c|c|}
\hline Method / Tested feature & OPTI-U & Multi-position & Comparative \\
\hline External cylinder diameter D [mm] & 34.518 & 34.562 & 34.548 \\
\hline Measurement uncertainty U [mm] & 0.167 & 0.211 & 0.133 \\
\hline Internal cylinder diameter d [mm] & 20.208 & 20.284 & 20.229 \\
\hline Measurement uncertainty U [mm] & 0.289 & 0.372 & 0.237 \\
\hline
\end{tabular}




\section{CONCLUSIONS}

The development of the uncertainty database is a promising project that can bring many benefits to the world of metrology and also to the widely understood industry where the necessity of measurements uncertainty determination is an important issue for even the most experienced operators. At the initial stage, work connected with collecting information (geometry/size of a measured object, measurement system i.e. maximum permissible error (MPE) of the machine, selection of a sensor, the number of operators, measurement strategy, external conditions) from particular measuring tasks is done by complementing records of the database. However it is possible the automation of this process based on a developed software monitoring the collection of measurement results data with calculated measurements uncertainties. Currently, tests are carried out for various geometry models made with the use of fused deposition modelling (FDM) method, using all optical measuring systems available in the Laboratory of Coordinate Metrology. In the future, the database will be developed with also contact and multisensory measurements.

\section{Acknowledgements}

Reported research were realized within confines of project financed by Polish National Centre for Research and Development No: LIDER/024/559/L-4/12/NCBR/2013 and by Ministry of Science and Higher Education M10/408/DS-M/2013

\section{REFERENCES}

1. Hansen H.N., Bariani P., De Chiffre L.: Modelling and Measurement Uncertainty Estimation for Integrated AFM-CMM Instrument. CIRP Annals - Manufacturing Technology, 54, 1, 2005, 531-534.

2. Jakubiec W., Płowucha W., Starczak M.: Analytical estimation of coordinate measurement uncertainty. Measurement, 45, 10, 2012, 2299-2308.

3. Gąska A., Sładek J., Ostrowska K., Kupiec R., Krawczyk M., Harmatys W., Gąska P., Gruza M., Szewczyk D., Knapik R., Kmita A.: Determination of coordinate measuring machines accuracy changes made by different nodes density in CAA matrix. XI International Symposium on Measurement and Quality Control, Cracow, Poland, 2013.
4. Ostrowska K., Gąska A., Sładek J.: Determining the uncertainty of measurement with the use of a Virtual Coordinate Measuring Arm. International Journal of Advanced Manufacturing Technology, 71, 1-4, 2014, 529-537.

5. Jakubiec W., Płowucha W.: First Coordinate Measurements Uncertainty Evaluation Software Fully Consistent with the GPS Philosophy. XII CIRP Conference on Computer Aided Tolerancing, Huddersfield, UK, 2012, 317-322.

6. Sładek J., Gąska A.: Evaluation of coordinate measurement uncertainty with use of virtual machine model based on Monte Carlo method. Measurement, 45, 2012, 1564-1575.

7. PN-EN ISO 15530-3:2011 Geometrical product specifications (GPS)- Coordinate measuring machines (CMM): Technique for determining the uncertainty of measurement - Part 3: Use of calibrated workpieces or measurement standards.

8. TS ISO 15530-2 Geometrical Product Specifications (GPS) Coordinate measuring machines (CMMs): Techniques for evaluation of the uncertainty of measurement - Use of multiple measurements strategies in the calibration of artefacts, 2005.

9. PN-EN ISO 10360-1:2003 Geometrical Product Specifications (GPS) - Acceptance and reverification tests for coordinate measuring machines (CMM) - Part 1: Vocabulary.

10. PN-EN ISO 14253-1 Geometrical Product Specifications (GPS) - Inspection by measurement of workpieces and measuring equipment - Part 1: Decision rules for proving conformance or nonconformance with specifications.

11. Sładek J.: Dokładność pomiarów współrzędnościowych (Accuracy of coordinate measurements). Cracow University of Technology, Cracow 2011.

12. Central Office of Measures Guide to the Expression of Uncertainty in Measurement (GUM) ISO $1993 / 1995$ with the addition to polish edition of J.M. Jaworski, Warsaw 1999.

13. Ostrowska K., Szewczyk D., Sładek J.: Determination of geometric elements measurement uncertainty with the use of optical system operating based on structured light. XI Coordinate Meausuring Technique, Bielsko-Biała, Poland, 2014.

14. Kunzmann H., Wäldele F., Wilkenin G., Corbett J., McKeown W., Hümmler J.: Progress in precision engineering and nanotechnology. t. 1. Hansen H.N., Trapet: An Approach to Uncertainty Estimation in Coordinate Metrology. Germany, 1997, 295-297.

15. Hansen H.N.: Verification and calibration of coordinate measuring machines. $\mathrm{Ph}$. D - thesis, Department of Manufacturing Engineering, Technical University of Denmark, 1999. 